\title{
LECTURES ON LINEAR STABILITY OF ROTATING BLACK HOLES
}

\author{
FELIX FINSTER
}

NOVEMBER 2018

\begin{abstract}
These lecture notes are concerned with linear stability of the nonextreme Kerr geometry under perturbations of general spin. After a brief review of the Kerr black hole and its symmetries, we describe these symmetries by Killing fields and work out the connection to conservation laws. The Penrose process and superradiance effects are discussed. Decay results on the long-time behavior of Dirac waves are outlined. It is explained schematically how the Maxwell equations and the equations for linearized gravitational waves can be decoupled to obtain the Teukolsky equation. It is shown how the Teukolsky equation can be fully separated to a system of coupled ordinary differential equations. Linear stability of the non-extreme Kerr black hole is stated as a pointwise decay result for solutions of the Cauchy problem for the Teukolsky equation. The stability proof is outlined, with an emphasis on the underlying ideas and methods.
\end{abstract}

\section{Contents}

1. Introduction

2. The Kerr Black Hole

3. Symmetries and Killing Fields

4. The Penrose Process and Superradiance

5. The Scalar Wave Equation in the Kerr Geometry

6. An Overview of Linear Wave Equations in the Kerr Geometry

6.1. The Dirac Equation

6.2. Massless Equations of General Spin, the Teukolsky Equation

7. Separation of the Teukolsky Equation

8. Results on Linear Stability and Superradiance

9. Hamiltonian Formulation and Integral Representations

10. A Spectral Decomposition of the Angular Teukolsky Operator

11. Invariant Disk Estimates for the Complex Riccati Equation

12. Separation of the Resolvent and Contour Deformations

13. Proof of Pointwise Decay

14. Concluding Remarks

References 


\section{INTRODUCTION}

These lectures are concerned with the black hole stability problem. Since this is a broad topic which many people have been working on, we shall restrict attention to specific aspects of this problem: First, we will be concerned only with linear stability. Indeed, the problem of nonlinear stability is much harder, and at present there are only few rigorous results. Second, we will concentrate on rotating black holes. This is because the angular momentum leads to effects (Penrose process, superradiance) which make the rotating case particularly interesting. Moreover, the focus on rotating black holes gives a better connection to my own research, which was carried out in collaboration with Niky Kamran (McGill), Joel Smoller (University of Michigan) and Shing-Tung Yau (Harvard). The linear stability result for general spin was obtained together with Joel Smoller (see [30] and the survey article [29]). Before beginning, I would like to remember Joel Smoller, who sadly passed away in September 2017. These notes are dedicated to his memory.

\section{The Kerr Black Hole}

In general relativity, space and time are combined to a four-dimensional spacetime, which is modelled mathematically by a Lorentzian manifold $(\mathscr{M}, g)$ of signature $(+---)$ (for more elementary or more detailed introductions to general relativity see the textbooks [1, 36, 44, 42]). The gravitational field is described geometrically in terms of the curvature of space-time. Newton's gravitational law is replaced by the Einstein equations

$$
R_{j k}-\frac{1}{2} R g_{j k}=8 \pi \kappa T_{j k}
$$

where $R_{j k}$ is the Ricci tensor, $R$ is scalar curvature, and $\kappa$ denotes the gravitational constant. Here $T_{j k}$ is the energy-momentum tensor which describes the distribution of matter in space-time.

A rotating black hole is described by the Kerr geometry. It is a solution of the vacuum Einstein equations discovered in 1963 by Roy Kerr. In the so-called BoyerLindquist coordinates, the Kerr metric takes the form (see [7, 37])

$$
d s^{2}=\frac{\Delta}{U}\left(d t-a \sin ^{2} \vartheta d \varphi\right)^{2}-U\left(\frac{d r^{2}}{\Delta}+d \vartheta^{2}\right)-\frac{\sin ^{2} \vartheta}{U}\left(a d t-\left(r^{2}+a^{2}\right) d \varphi\right)^{2},
$$

where

$$
U=r^{2}+a^{2} \cos ^{2} \vartheta, \quad \Delta=r^{2}-2 M r+a^{2},
$$

and the coordinates $(t, r, \vartheta, \varphi)$ are in the range

$$
-\infty<t<\infty, \quad M+\sqrt{M^{2}-a^{2}}<r<\infty, \quad 0<\vartheta<\pi, \quad 0<\varphi<2 \pi .
$$

The parameters $M$ and $a M$ describe the mass and the angular momentum of the black hole.

In the case $a=0$, one recovers the Schwarzschild metric

$$
d s^{2}=\left(1-\frac{2 M}{r}\right) d t^{2}-\left(1-\frac{2 M}{r}\right)^{-1} d r^{2}-r^{2}\left(d \theta^{2}+\sin ^{2} \theta d \varphi^{2}\right) .
$$

In this case, the function $\Delta$ has two roots

$$
\begin{array}{ll}
r=2 M & \text { event horizon } \\
r=0 & \text { curvature singularity . }
\end{array}
$$


In the region $r>2 M$, the so-called exterior region, $t$ is a time coordinate, whereas $r, \vartheta$ and $\varphi$ are spatial coordinates. More precisely, $(\vartheta, \varphi)$ are polar coordinates, whereas the radial coordinate $r$ is determined by the fact that the two-surface $S=\left\{t=t_{0}, r=r_{0}\right\}$ has area $4 \pi r_{0}^{2}$. The region $r<2 M$, on the other hand, is the interior region. In this region, the radial coordinate $r$ is time, whereas $t$ is a spatial coordinate. Since time always propagates to the future, the event horizon can be regarded as the "boundary of no escape." The surface $r=2 M$ merely is a coordinate singularity of our metric. This becomes apparent by transforming to Eddington-Finkelstein or Kruskal coordinates. For brevity, we shall not enter the details here.

In the case $a \neq 0$, the singularity structure is more involved. The function $U$ is always strictly positive. The function $\Delta$ has the two roots

$$
\begin{array}{ll}
r_{0}:=M+\sqrt{M^{2}-a^{2}} & \text { event horizon } \\
r_{1}:=M-\sqrt{M^{2}-a^{2}} & \text { Cauchy horizon . }
\end{array}
$$

If $a^{2}>M^{2}$, these roots are complex. This corresponds to the unphysical situation of a naked singularity. We shall not discuss this case here, but only consider the so-called

$$
\text { non-extreme case } \quad M^{2}<a^{2} \text {. }
$$

In this case, the hypersurface

$$
r=r_{1}:=M+\sqrt{M^{2}-a^{2}}
$$

again defines the event horizon of the black hole. In what follows, we shall restrict attention to the exterior region $r>r_{1}$. This is because classically, no information can be transmitted from the interior of the black hole to its exterior. Therefore, it is impossible for principal reasons to know what happens inside the black hole. With this in mind, it seems pointless to study the black hole inside the event horizon, because this study will never be tested or verified by experiments.

We finally remark that in quantum gravity, the situation is quite different because it is conceivable that a black hole might "evaporate," in which case the interior of the black hole might become accessible to observations. In physics, such questions are often discussed in connection with the so-called information paradox, which states that the loss of information at the event horizon is not compatible with the unitary time evolution in quantum theory. I find such questions related to quantum effects of a black hole quite interesting, and indeed most of my recent research is devoted to quantum gravity (in an approach called causal fermion systems; see for example the textbook [13] or the survey paper [20]). But since this summer school is devoted to classical gravity, I shall not enter this topic here.

\section{Symmetries and Killing Fields}

The Kerr geometry is stationary and axisymmetric. This is apparent in BoyerLindquist coordinates (2.2) because the metric coefficients are

$$
\begin{array}{ll}
\text { independent of } t: & \text { stationary } \\
\text { independent of } \varphi: & \text { axisymmetric } .
\end{array}
$$

These symmetries can be described more abstractly using the notion of Killing fields. We recall how this works because we need it later for the description of the Penrose process and superradiance. We restrict attention to the time translation symmetry, because for for the axisymmetry or other symmetries, the argument is similar. 
Given $\tau \in \mathbb{R}$, we consider the mapping

$$
\Phi_{\tau}: \mathscr{M} \rightarrow \mathscr{M}, \quad(t, x) \mapsto(t+\tau, x)
$$

(where $x$ stands for the spatial coordinates $(r, \vartheta, \varphi)$ ). The fact that the metric coefficients are time independent means that $\Phi_{\tau}$ is an isometry, defined as follows. The derivative of $\Phi_{\tau}$ (i.e. the linearization; it is sometimes also denoted by $\left.\left(\Phi_{\tau}\right)_{*}\right)$ is a mapping between the corresponding tangent spaces,

$$
\left.D \Phi_{\tau}\right|_{p}: T_{p} \mathscr{M} \rightarrow T_{\Phi_{\tau}(x)} \mathscr{M}
$$

Being an isometry means that

$$
g_{p}(u, v)=g_{\Phi_{\tau}(p)}\left(\left.D \Phi_{\tau}\right|_{p} u,\left.D \Phi_{\tau}\right|_{p} v\right) \quad \text { for all } u, v \in T_{p} \mathcal{M} .
$$

Let us evaluate this equation infinitesimally in $\tau$. We first introduce the vector field $K$ by

$$
K:=\left.\frac{d}{d \tau} \Phi_{\tau}\right|_{\tau=0} .
$$

Choosing local coordinates, we obtain in components

$$
\left(\left.D \Phi_{\tau}\right|_{p} u\right)^{a}=\frac{\partial \Phi_{\tau}^{a}(p)}{\partial x^{i}} u^{i},
$$

where for clarity we denote the tensor indices at the point $\Phi_{\tau}(x)$ by $a$ and $b$. We then obtain

$$
\begin{aligned}
0 & =\left.\frac{d}{d \tau} g_{\Phi_{\tau}(p)}\left(\left.D \Phi_{\tau}\right|_{p} u,\left.D \Phi_{\tau}\right|_{p} v\right)\right|_{\tau=0} \\
& =\left.\frac{d}{d \tau}\left(g_{a b}\left(\Phi_{\tau}(p)\right) \frac{\partial \Phi_{\tau}^{a}(p)}{\partial x^{i}} u^{i} \frac{\partial \Phi_{\tau}^{b}(p)}{\partial x^{j}} v^{j}\right)\right|_{\tau=0} \\
& =\partial_{k} g(u, v) K^{k}+g\left(u^{i} \partial_{i} K, v\right)+g\left(u, v^{j} \partial_{j} K\right) .
\end{aligned}
$$

Choosing Gaussian coordinates at $p$, one sees that this equation can be written covariantly as

$$
0=g\left(\nabla_{u} K, v\right)+g\left(u, \nabla_{v} K\right)
$$

where $\nabla$ is the Levi-Civita connection. This is the Killing equation, which can also be written in the shorter form

$$
0=\nabla_{(i} K_{j)}:=\frac{1}{2}\left(\nabla_{i} K_{j}+\nabla_{j} K_{i}\right) .
$$

A vector field which satisfies the Killing equation is referred to as a Killing field. We remark that if the flow lines exist on an interval containing zero and $\tau$, then the resulting diffeomorphism $\Phi_{\tau}$ is indeed an isometry of $\mathscr{M}$.

A variant of Noether's theorem states that Killing symmetries, which describe infinitesimal symmetries of space-time, give rise to corresponding conservation laws. For geodesics, these conservation laws are obtained simply by taking the Lorentzian inner product of the Killing vector field and the velocity vector of the geodesic. Indeed, let $\gamma(\tau)$ be a parametrized geodesic, i.e.

$$
\nabla_{\tau} \dot{\gamma}(\tau)=0
$$

Then, denoting the metric for simplicity by $\langle., .\rangle_{p}:=g_{p}(.,$.$) , we obtain$

$$
\begin{aligned}
& \frac{d}{d \tau}\langle K(\gamma(\tau)), \dot{\gamma}(\tau)\rangle_{\gamma(\tau)}=\left\langle\nabla_{\tau} K(\gamma(\tau)), \dot{\gamma}(\tau)\right\rangle_{\gamma(\tau)}+\left\langle K(\gamma(\tau)), \nabla_{\tau} \dot{\gamma}(\tau)\right\rangle_{\gamma(\tau)} \\
& \quad=\left\langle\nabla_{\tau} K(\gamma(\tau)), \dot{\gamma}(\tau)\right\rangle_{\gamma(\tau)}=\left.\nabla_{i} K_{j}\right|_{\gamma(\tau)} \dot{\gamma}^{i}(\tau) \dot{\gamma}^{j}(\tau)=0
\end{aligned}
$$



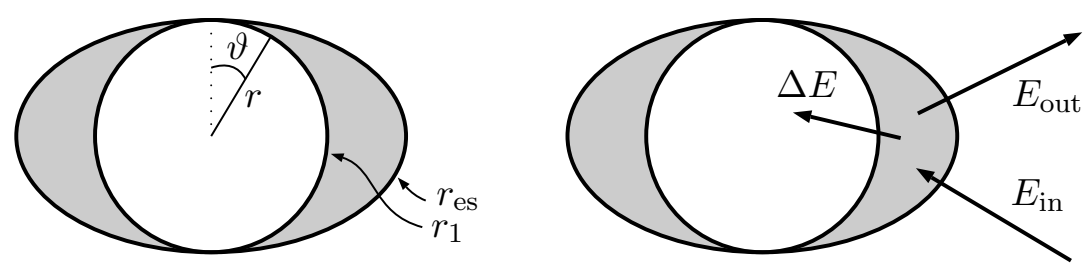

Figure 1. Schematic picture of the ergosphere (left) and the Penrose process (right).

where in the last step we used the Killing equation (3.1). We thus obtain the conservation law

$$
\langle K(\gamma(\tau)), \dot{\gamma}(\tau)\rangle_{\gamma(\tau)}=\text { const }
$$

which holds for any parametrized geodesic $\gamma(\tau)$ and any Killing field $K$.

\section{The Penrose Process and Superradiance}

In the Kerr geometry, the two vector fields $\partial_{t}$ and $\partial_{\varphi}$ are Killing fields. The corresponding conserved quantities are

$$
\begin{array}{ll}
E:=\left\langle\frac{\partial}{\partial t}, \dot{\gamma}(\tau)\right\rangle_{\gamma(\tau)} & \text { energy } \\
A:=\left\langle\frac{\partial}{\partial \varphi}, \dot{\gamma}(\tau)\right\rangle_{\gamma(\tau)} & \text { angular momentum . }
\end{array}
$$

Let us consider the energy in more detail for a test particle moving along the geodesic $\gamma$. In this case, $\gamma(\tau)$ is a causal curve (i.e. $\dot{\gamma}(\tau)$ is timelike or null everywhere), and we always choose the parametrization such that $\gamma$ is future-directed (i.e. the time coordinate $\gamma^{0}(\tau)$ is monotone increasing in $\tau$ ). In the asymptotic end (i.e. for large $r$ ), the Killing field $\partial_{t}$ is timelike and future-directed. As a consequence, the inner product in (4.1) is strictly positive. This corresponds to the usual concept of the energy being a non-negative quantity. We point out that this result relies on the assumption that the Killing field $\partial_{t}$ is timelike. However, if this Killing field is spacelike, then the inner product in (4.1) could very well be negative. In order to verify if this case occurs, we compute

$$
\left\langle\frac{\partial}{\partial t}, \frac{\partial}{\partial t}\right\rangle=g_{00}=\frac{\Delta}{U}-\frac{a^{2} \sin ^{2} \vartheta}{U}=\frac{1}{U}\left(r^{2}-2 M r+a^{2} \cos ^{2} \vartheta\right),
$$

where we read off the corresponding metric coefficient in (2.2) and simplified it using (2.3). Computing the roots, one sees that the Killing field $\partial_{t}$ indeed becomes null on the surface

$$
r=r_{\mathrm{es}}:=M+\sqrt{M^{2}-a^{2} \cos ^{2} \vartheta}
$$

the so-called ergosphere. Comparing with the formula for the event horizon (2.4), one sees that the ergosphere is outside the event horizon and intersects the event horizon at the poles $\vartheta=0, \pi$ (see the left of Figure11). The region $r_{1}<r<r_{\mathrm{es}}$ is the so-called ergoregion.

The ergosphere causes major difficulties in the proof of linear stability of the Kerr geometry. These difficulties are not merely technical, but they are related to physical phenomena, as we now explain step by step. The name ergosphere is motivated from the fact that it gives rise to a mechanism for extracting energy from a rotating black hole. This effect was first observed by Roger Penrose [38] and is therefore referred 
to as the Penrose process. In order to explain this effect, we consider a spaceship of energy $E_{\text {in }}$ which flies into the ergoregion (see the right of Figure 1), where it ejects a projectile of energy $\Delta E$ which falls into the black hole. After that, the spaceship flies out of the ergoregion with energy $E_{\text {out }}$. Due to energy conservation, we know that $E_{\text {in }}=E_{\text {out }}+\Delta E$. By choosing the momentum of the projectile appropriately, one can arrange that the energy $\Delta E$ is negative. Then the final energy $E_{\text {out }}$ is larger than the initial energy $E_{\text {in }}$, which means that we gained energy. This energy gain does not contradict total energy conservation, because one should think of the energy as being extracted from the black hole (this could indeed be made precise by taking into account the back reaction of the space ship onto the black hole, but we do not have time for entering such computations). Therefore, the Penrose process is similar to the so-called "swing-by" or "gravitational slingshot," where a satellite flies close to a planet of our solar system and uses the kinetic energy of the planet for its own acceleration. The surprising effect is that in the Penrose process, one can extract energy from the black hole, although the matter of the black hole is trapped behind the event horizon.

The wave analogue of the Penrose process is called superradiance. Instead of the spaceship one considers a wave packet flying in the direction of the black hole. The wave propagates as described by a corresponding wave equation (we will see such wave equations in more detail later). As a consequence, part of the wave will "fall into" the black hole, whereas the remainder will pass the black hole and will eventually leave the black hole region. If the energy of the outgoing wave is larger than the energy of the oncoming wave, then one speaks of superradiant scattering. This effect is quite similar to the Penrose process. However, one major difference is that, in contrast to the Penrose process, there is no freedom in choosing the momentum of the projectile. Instead, the dynamics is determined completely by the initial data, so that the only freedom is to prepare the incoming wave packet. As we shall see later in this lecture, superradiance indeed occurs for scalar waves in the Kerr geometry.

\section{The Scalar Wave Equation in the Kerr Geometry}

In preparation of the analysis of general linear wave equations, we begin with the simplest example: the scalar wave equation. It has the useful property that it is of variational form, meaning that it can be derived from an action principle. Indeed, choosing the Dirichlet action

$$
\mathcal{S}=\int_{\mathscr{M}} g^{i j}\left(\partial_{i} \phi\right)\left(\partial_{j} \phi\right) d \mu_{\mathscr{M}},
$$

(where $d \mu_{\mathcal{M}}=\sqrt{|\operatorname{det} g|} d^{4} x$ is the volume measure induced by the Lorentzian metric), demanding criticality for first variations gives the scalar wave equation

$$
0=\square \phi:=\nabla_{i} \nabla^{i} \phi .
$$

The main advantage of the variational formulation is that Noether's theorem relates symmetries to conservation laws. Another method for getting these conservation laws, which is preferable to us because it is closely related to the notion of Killing fields, is to work directly with the energy-momentum tensor of the field. Recall that in the Einstein equations (2.1), the Einstein tensor on the left is divergence-free as a consequence of the second Bianchi identities. Therefore, the energy-momentum tensor is also divergence-free,

$$
\nabla^{i} T_{i j}=0
$$




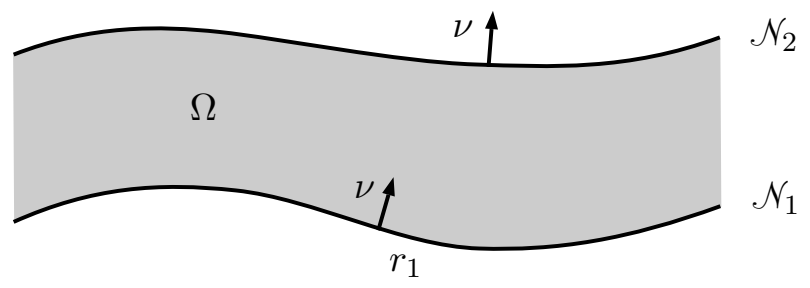

Figure 2. Conservation law corresponding to a Killing symmetry.

Now let $K$ be a Killing field. Contracting the energy-momentum tensor with the Killing field gives a vector field,

$$
u^{i}:=T^{i j} K_{j} .
$$

The calculation

$$
\nabla_{i} u^{i}=\left(\nabla_{i} T^{i j}\right) K_{j}+T^{i j} \nabla_{i} K_{j}=0
$$

(where the first summand vanishes according to the conservation law (5.1), whereas the second summand is zero in view of the Killing equation (3.1) and the symmetry of the energy-momentum tensor) shows that this vector field is divergence-free. Therefore, integrating the divergence of $u$ over a space-time region $\Omega$ and using the Gauß divergence theorem, we conclude that the flux integral of $u$ through the surface $\partial \Omega$ vanishes. The situation we have in mind is that the set $\Omega$ is the region between two spacelike hypersurfaces $\mathcal{N}_{1}$ and $\mathcal{N}_{2}$ (see Figure 2). Assuming that the vector field $u$ has suitable decay properties at spatial infinity (in the simplest case that it has spatially compact support), we obtain the conservation law

$$
0=\int_{\Omega} \nabla_{i} u^{i} d \mu_{\mathcal{M}}=\int_{\mathcal{N}_{1}} T_{i j} \nu^{i} K^{j} d \mu_{\mathcal{N}_{1}}-\int_{\mathcal{N}_{1}} T_{i j} \nu^{i} K^{j} d \mu_{\mathcal{N}_{2}},
$$

where $\nu$ is the future-directed normal on $\mathcal{N}_{1 / 2}$ and $d \mu_{\mathcal{N}_{1 / 2}}$ is the volume measure corresponding to the induced Riemannian metric.

In the Kerr geometry in Boyer-Lindquist coordinates, the Dirichlet action takes the explicit form

with

$$
\mathcal{S}=\int_{-\infty}^{\infty} d t \int_{r_{1}}^{\infty} d r \int_{-1}^{1} d(\cos \vartheta) \int_{0}^{\pi} d \varphi \mathcal{L}(\phi, \nabla \phi)
$$

$$
\begin{aligned}
\mathcal{L}(\phi, \nabla \phi)= & -\Delta\left|\partial_{r} \phi\right|^{2}+\frac{1}{\Delta}\left|\left(\left(r^{2}+a^{2}\right) \partial_{t}+a \partial_{\varphi}\right) \phi\right|^{2} \\
& -\sin ^{2} \vartheta\left|\partial_{\cos \vartheta} \phi\right|^{2}-\frac{1}{\sin ^{2} \vartheta}\left|\left(a \sin ^{2} \vartheta \partial_{t}+\partial_{\varphi}\right) \phi\right|^{2} .
\end{aligned}
$$

Considering first variations, the scalar wave equation becomes

$$
\begin{aligned}
& {\left[\frac{\partial}{\partial r} \Delta \frac{\partial}{\partial r}-\frac{1}{\Delta}\left\{\left(r^{2}+a^{2}\right) \frac{\partial}{\partial t}+a \frac{\partial}{\partial \varphi}\right\}^{2}\right.} \\
& \left.\quad+\frac{\partial}{\partial \cos \vartheta} \sin ^{2} \vartheta \frac{\partial}{\partial \cos \vartheta}+\frac{1}{\sin ^{2} \vartheta}\left\{a \sin ^{2} \vartheta \frac{\partial}{\partial t}+\frac{\partial}{\partial \varphi}\right\}^{2}\right] \phi=0 .
\end{aligned}
$$

Using the formula for the energy-momentum tensor

$$
T_{i j}=\left(\partial_{i} \phi\right)\left(\partial_{j} \phi\right)-\frac{1}{2}\left(\partial_{k} \phi\right)\left(\partial^{k} \phi\right) g_{i j},
$$



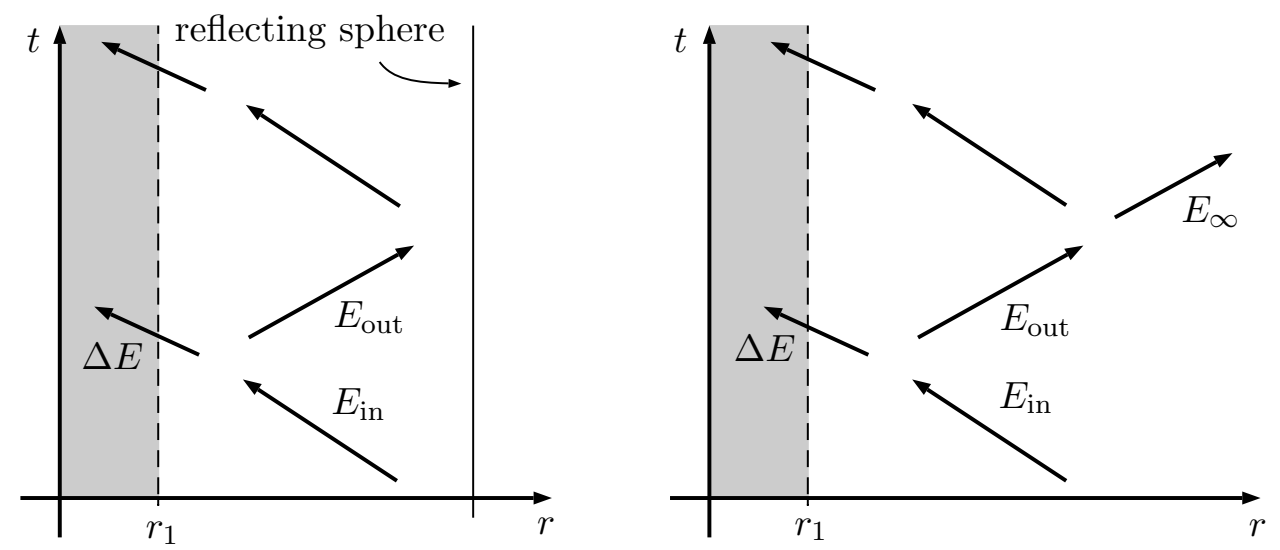

Figure 3. The black hole bomb (left) and wave propagation in the Kerr geometry (right).

the conserved energy becomes

$$
\begin{aligned}
E & :=\int_{\mathcal{N}_{t}} T_{i j} \nu^{j}\left(\partial_{t}\right)^{j} d \mu_{\mathcal{N}_{t}}=\int_{\mathcal{N}_{t}} T_{i 0}\left(\partial_{t}\right)^{j} d \mu_{\mathcal{N}_{t}} \\
& =\int_{r_{1}}^{\infty} d r \int_{-1}^{1} d(\cos \vartheta) \int_{0}^{2 \pi} d \varphi \mathcal{E}
\end{aligned}
$$

with the "energy density"

$$
\begin{aligned}
\mathcal{E}= & \left(\frac{\left(r^{2}+a^{2}\right)^{2}}{\Delta}-a^{2} \sin ^{2} \vartheta\right)\left|\partial_{t} \phi\right|^{2}+\Delta\left|\partial_{r} \phi\right|^{2} \\
& +\sin ^{2} \vartheta\left|\partial_{\cos \vartheta} \phi\right|^{2}+\left(\frac{1}{\sin ^{2} \vartheta}-\frac{a^{2}}{\Delta}\right)\left|\partial_{\varphi} \phi\right|^{2} .
\end{aligned}
$$

Using (2.3), one sees that the factor in front of the term $\left|\partial_{\varphi} \phi\right|$ is everywhere positive. However, the factor in front of the term $\left|\partial_{t} \phi\right|^{2}$ is negative precisely inside the ergosphere (4.3). This consideration shows that, exactly as for point particles (4.1), the energy of scalar waves may again be negative inside the ergosphere.

What does the indefiniteness of the energy tell us? We first point out that it does not imply that superradiance really occurs, because in order to analyze superradiance, one must study the dynamics of waves. Instead, it only means that there is a possibility for superradiance to occur. In technical terms, the indefiniteness of the energy leads to the difficulty that energy conservation does not give us control of the Sobolev norm of the wave. A possible scenario, which does not contradict energy conservation, is that the amplitude of the wave grows in time both inside and outside the the ergosphere. It is a major task in proving linear stability to rule out this scenario.

The basic difficulty can be understood qualitatively in more detail in the scenario of the so-called black hole bomb as introduced by Press and Teukolsky [39] and studied by Cardoso et al [5]. In this gedanken experiment, one puts a metal sphere around a Kerr black hole (as shown schematically on the left of Figure 3. We consider a wave packet of energy $E_{\text {in }}$ inside the sphere flying towards the black hole. Part of the wave will cross the event horizon, while the remainder will pass the black hole. As in the above description of superradiance, we assume that the energy $\Delta E$ of the wave crossing the event horizon is negative. Then the energy $E_{\text {out }}$ of the outgoing wave is larger than the 
energy $E_{\text {in }}$ of the incoming wave. The outgoing wave is reflected on the metal sphere, becoming a new wave which again flies towards the black hole. If it can be arranged that the new incoming wave has the same shape as the original wave, this process repeats itself, generating in each step a certain positive energy. In this scenario, the energy density inside the metal sphere would grow exponentially fast in time. When the energy density gets too large, the metal sphere would explode, explaining the name "black hole bomb." For clarity, we point out that in this mechanism one always assumes that the total energy extracted from the black hole is much smaller than the total rotational energy of the black hole, so that the back reaction on the black hole need not be taken into account.

The black hole bomb suggests that, putting a metal sphere around the black hole could lead to an instability, which would become manifest in an explosion of the metal sphere. The point of interest in connection to the stability problem for rotating black holes is that a very similar scenario might occur even without the metal sphere: We again consider a wave packet flying towards the black hole. Again, part of the wave with energy $\Delta E$ crosses the event horizon, whereas the remainder of energy $E_{\text {out }}$ passes the black hole. The point is that only part of this wave will reach null infinity. Another part will be backscattered by the gravitational field and will again fly towards the black hole. Therefore, except for the "energy loss" $E_{\infty}$ by the part of the wave propagating to null infinity, we are again in the scenario of the black hole bomb where the process repeats itself, potentially leading to an exponential increase in time of the amplitude of the wave.

Clearly, this picture is oversimplified because, instead of wave packets, one must consider waves which are spread out in space, leading to a nonlocal problem. Nevertheless, in this picture it becomes clear why the problem of linear stability of rotating black holes amounts to a quantitative question: Can the initial wave packet be arranged such that the "energy gain" $-\Delta E$ is larger than the "energy loss" $E_{\infty}$ ? If the answer is yes, a rotating black hole should be unstable, and it should decay by

radiation of gravitational waves to a Schwarzschild black hole. It is the main goal of these lectures to explain why this does not happen, i.e. why rotating black holes are linearly stable. Before we can enter this problem in mathematical detail, we need to introduce linear wave equations and review a few structural results.

\section{An Overview of Linear Wave Equations in the Kerr Geometry}

6.1. The Dirac Equation. In these lectures I shall not enter the details of the Dirac equation, although most of my work has been concerned with or related to the Dirac equation. I only want to explain why the analysis for the Dirac equation is much easier than for other wave equations.

The Dirac equation describes a relativistic quantum mechanical particle with spin. In order to keep the setting as simple as possible, we work in coordinates and local trivializations of the spinor bundle (which has the advantage that we do not need to even define what the spinor bundle is). Then the Dirac wave function $\psi(x) \in \mathbb{C}^{4}$ has four complex components, which describe the spinorial degrees of freedom of the wave function. The Dirac equation reads

$$
\left(i \gamma^{j}(x) \partial_{j}+\mathcal{B}-m\right) \psi=0
$$


Here $m$ is the rest mass of the Dirac particles, and the four matrices $\gamma^{j}$ encode the Lorentzian metric via the anti-commutation relations

$$
\left\{\gamma^{j}(x), \gamma^{k}(x)\right\}=2 g^{j k}(x) \mathbb{1}_{\mathbb{C}^{4}},
$$

where the anti-commutator is defined by

$$
\left\{\gamma^{j}, \gamma^{k}\right\}:=\gamma^{j} \gamma^{k}+\gamma^{k} \gamma^{j}
$$

The multiplication operator $\mathcal{B}$ involves the so-called spin coefficients, which, in analogy to the Christoffel symbols of the Levi-Civita connection, are formed of first partial derivatives of the Dirac matrices. We do not need the details here and refer instead to the explicit formulas in [12] or [21, Chapter 3].

Coming from quantum mechanics, the Dirac equation has additional structures which allow for the probabilistic interpretation of the wave function. In particular, there is a quantity which can be interpreted as the probability density as seen by an observer, and the spatial integral of this probability density is equal to one, for any fixed time of the observer. This probability integral is described mathematically as follows. The spinors at a space-time point $x \in \mathcal{M}$ are endowed with an indefinite inner product of signature $(2,2)$, which we denote by $\prec \psi \mid \psi \succ_{x}$. For any solution $\psi$ of the Dirac equation, the pointwise expectation value of the Dirac matrices with respect to this inner product defines a vector field

$$
J^{k}(x):=\prec \psi(x) \mid \gamma^{k} \psi(x) \succ_{x} .
$$

This vector field is the so-called Dirac current. The structure of the Dirac equation ensures that this vector field is always non-spacelike and future-directed. Moreover, as a consequence of the Dirac equation, this vector field is divergence-free, i.e.

$$
\nabla_{k} J^{k}(x)=0
$$

(where $\nabla$ is again the Levi-Civita connection); this is referred to as current conservation. Integrating this equation over a region $\Omega$ between two spacelike hypersurfaces (as shown in Figure 2), one obtains the conservation law

$$
\int_{\mathcal{N}_{2}} J_{i} \nu^{i} d \mu_{\mathcal{N}_{2}}=\int_{\mathcal{N}_{1}} J_{i} \nu^{i} d \mu_{\mathcal{N}_{1}}
$$

(here we again assume that the Dirac wave function has suitable decay properties at spatial infinity). In view of this conservation law and the linearity of the Dirac equation, one can normalize the Dirac solutions such that the integral in (6.1) equals one. Then the integrand in (6.1) has the interpretation as the probability density for an observer for whom the spacelike hypersurface $\mathcal{N}_{1}\left(\right.$ or $\left.\mathcal{N}_{2}\right)$ describes space. We point out that the probability density is non-negative simply as a consequence of the fact that the current vector is non-spacelike and future-directed, and that the normal is timelike and future-directed. In particular, the probability density is non-negative even inside the ergosphere; note also that, in contrast to (5.2), the integrand in (6.1) does not involve a Killing field.

These structures coming from the probabilistic interpretation of the Dirac equation are a major simplification for analyzing the long-time dynamics of Dirac waves in the Kerr geometry. Namely, the current integral (6.1) can be used to define a scalar product on the solutions of the Dirac equation by

$$
(\psi \mid \phi)_{t}:=\int_{N_{t}} \prec \psi \mid \gamma^{j} \phi \succ_{x} \nu_{j} d \mu_{\mathcal{N}_{t}}
$$


where $\mathcal{N}_{t}$ is the surface of constant time in Boyer-Lindquist coordinates outside the event horizon, and where we restrict attention to Dirac solutions with suitable decay properties on the event horizon and near spatial infinity (for example wave functions with spatially compact support outside the event horizon). Taking the completion, we obtain the Hilbert space $\left(\mathcal{H}_{t},(. \mid .)_{t}\right)$ of Dirac solutions. The conservation law (6.1) means that the time evolution operator $U_{t, t_{0}}: \mathcal{H}_{t_{0}} \rightarrow \mathcal{H}_{t}$ from time $t_{0}$ to time $t$ is a unitary operator. Since the Kerr geometry is stationary, we can canonically identify the Hilbert space $\mathcal{H}_{t}$ with $\mathcal{H}_{t_{0}}$ by time translation of the wave functions. Moreover, the unitary time evolution can be written as

$$
U_{t, t_{0}}=e^{-i\left(t-t_{0}\right) H},
$$

where the so-called Dirac Hamiltonian $H$ is a self-adjoint operator on the Hilbert space (the self-adjointness extension can be constructed in general using Stone's theorem or Chernoff's method [8]). In this way, the long-time dynamics can be related to spectral properties of a self-adjoint operator on a Hilbert space. No superradiance phenomena occur.

More details on the Dirac equation and the above method can be found in my joint papers with Niky Kamran, Joel Smoller and Shing-Tung Yau [14, 16, 15]. For the general method of constructing self-adjoint extensions, the more recent paper [22] may be useful.

6.2. Massless Equations of General Spin, the Teukolsky Equation. After the short excursion to quantum mechanics, we now return to classical waves. The waves of interest are

$$
\left\{\begin{array}{c}
\text { scalar waves } \\
\text { electromagnetic waves } \\
\text { gravitational waves }
\end{array}\right.
$$

Scalar waves were already considered in Section 5 they are studied mainly because of their mathematical simplicity. The waves of physical interest are electromagnetic and gravitational waves. Note that all these waves are massless.

All the above wave equations can be described in a unified framework due to Teukolsky [43]. We now explain schematically how the Teukolsky formulation works. Since the involved computations are quite lengthy, we cannot enter the details but refer instead to the textbook [7]. The Teukolsky equation is derived in the Newman-Penrose formalism, which we now briefly introduce. In this formalism, one works with a double null frame, i.e. with a set of vectors $(l, n, m, \bar{m})$ of the complexified tangent space with inner products

$$
\langle l, n\rangle=1, \quad\langle m, \bar{m}\rangle=-1,
$$

whereas all other inner products vanish,

$$
\langle l, l\rangle=\langle n, n\rangle=\langle m, m\rangle=\langle\bar{m}, \bar{m}\rangle=0 .
$$

In the example of Minkowski space in Cartesian coordinates $(t, x, y, z)$, one can choose $l$ and $n$ as two null vectors, for example

$$
l=\frac{1}{\sqrt{2}}\left(\frac{\partial}{\partial t}+\frac{\partial}{\partial x}\right) \quad \text { and } \quad n=\frac{1}{\sqrt{2}}\left(\frac{\partial}{\partial t}-\frac{\partial}{\partial x}\right) .
$$

The orthogonal complement of these two vectors is the two-dimensional spacelike plane spanned by the vectors $\partial_{y}$ and $\partial_{z}$. Therefore, the only way to obtain additional null 
vectors is to complexify by choosing for example

$$
m=\frac{1}{\sqrt{2}}\left(\frac{\partial}{\partial y}+i \frac{\partial}{\partial z}\right) \quad \text { and } \quad \bar{m}=\frac{1}{\sqrt{2}}\left(\frac{\partial}{\partial y}-i \frac{\partial}{\partial z}\right) .
$$

Likewise, on a Lorentzian manifold, the vectors $(l, n, m, \bar{m})$ form a basis of the complexified tangent space. The Lorentzian inner product $\langle.,$.$\rangle is extended to the complexified$ tangent space as a bilinear form (not sesquilinear; thus no complex conjugation is involved). The double null frame is well-suited for the analysis of the vacuum Einstein equations (indeed, the Kerr solution was discovered in the Newman-Penrose formalism).

Next, one combines the tensor components in the double null frame in complexvalued functions. In the example of the Maxwell field, this works as follows. The electromagnetic field tensor $F_{i j}$ has six real components (three electric and three magnetic field components). One combines these six real components to the three complex functions

$$
\Psi_{0}=F_{l m}, \quad \Psi_{1}=\frac{1}{2}\left(F_{l n}+F_{m \bar{m}}\right), \quad \Psi_{2}=F_{n \bar{m}} .
$$

Then the homogeneous Maxwell equations

$$
d F=0 \quad \text { and } \quad \nabla^{k} F_{j k}=0
$$

give rise to a first-order system of partial differential equations for $\Psi=\left(\Psi_{0}, \Psi_{1}, \Psi_{2}\right)$. For the gravitational field, one considers similarly the Weyl tensor $C_{i j k l}$. Linearized gravitational waves are described by linear perturbations of the Weyl tensor in the Newman-Penrose frame. Denoting the linear perturbation of the Weyl tensor by $W$, its ten real components are combined to the five complex functions

$$
\begin{gathered}
\Psi_{0}=-W_{l m l m}, \quad \Psi_{1}=-W_{l n l m}, \quad \Psi_{2}=-W_{l m \bar{m} n} \\
\Psi_{3}=-W_{l n \bar{m} n}, \quad \Psi_{4}=-W_{l \bar{m} n \bar{m}} .
\end{gathered}
$$

Linearizing the second Bianchi identities $R_{i j(k l ; m)}=0$ gives a first-order system of partial differential equations for $\Psi=\left(\Psi_{0}, \ldots, \Psi_{4}\right)$. In this formulation, the connection to the spin can be obtained simply by counting the number of degrees of freedom. In quantum mechanics, a wave function of spin $s$ has $2 s+1$ complex components. Therefore, we obtain the correct number of degrees of freedom if we set

$$
\begin{aligned}
\text { electromagnetic waves: } & \text { spin } s=1 \\
\text { gravitational waves: } & \operatorname{spin} s=2 .
\end{aligned}
$$

We remark that the connection to the spin is more profound than merely counting the number of degrees of freedom, but we have no time to explain how this works. For our purposes, it suffices to take the spin $s$ as a parameter which characterizes the massless wave equation by counting the number of components of the Newman-Penrose wave function $\Psi=\left(\Psi_{0}, \ldots, \Psi_{2 s}\right)$.

We write the first-order system of partial differential equations for electromagnetic waves or linearized gravitational waves symbolically as

$$
\mathcal{D}\left(\begin{array}{c}
\Psi_{0} \\
\vdots \\
\Psi_{2 s}
\end{array}\right)=0 .
$$

Working with this first-order system is not convenient for larger spin because the number of equations gets large, and the equations are coupled in a complicated way. 
But, as discovered by Teukolsky, the system of equations can be decoupled such as to obtain a second-order partial differential equation for one complex-valued function. This decoupling works schematically as follows: One chooses a Newman-Penrose null frame where $l$ and $n$ are aligned with the repeated principal null directions of the Weyl tensor (in this frame, the Newman-Penrose components of the Weyl tensor satisfy the equations $\psi_{0}=\psi_{1}=\psi_{3}=\psi_{4}=0$, with $\psi_{2}$ being the only non-zero component). Multiplying the linear first-order system (6.4) in this frame by a suitable first-order differential operator $D$, we obtain the equation

$$
0=D \mathcal{D}\left(\begin{array}{c}
\Psi_{0} \\
\vdots \\
\Psi_{2 s}
\end{array}\right)=\left(\begin{array}{ccccc}
T_{0} & 0 & \cdots & 0 & 0 \\
* & * & \cdots & * & * \\
\vdots & \vdots & \ddots & \vdots & \vdots \\
* & * & \cdots & * & * \\
0 & 0 & \cdots & 0 & T_{2 s}
\end{array}\right)\left(\begin{array}{c}
\Psi_{0} \\
\Psi_{1} \\
\vdots \\
\Psi_{2 s-1} \\
\Psi_{2 s}
\end{array}\right),
$$

where the stars stand for differential operators which we do not need to specify here. The point is that this procedure generates zeros in the first and last row of the matrix, giving rise to decoupled equations for the first and and last components of the NewmanPenrose wave function $\Psi$,

$$
T_{0} \Psi_{0}=0=T_{2 s} \Psi_{2 s} .
$$

Once the solution $\Psi_{0}$ or $\Psi_{2 s}$ is known, all the other components of $\Psi$ can be obtained by employing the so-called Teukolsky-Starobinsky identities, which have similarities to the "ladder operator" for the harmonic oscillator used for obtaining the excited states from the ground state. With this in mind, in what follows we restrict attention to the equations for $\Psi_{0}$ or $\Psi_{2 s}$ in (6.5). After detailed computations for the electromagnetic field and for linearized gravitational fields, in both cases one ends up with the same equation, except for a parameter $s$ describing the spin. We thus obtain the Teukolsky equation (sometimes called Teukolsky master equation; we use the form of the equation as given in [45])

$$
\begin{aligned}
\left(\frac{\partial}{\partial r}\right. & \Delta \frac{\partial}{\partial r}-\frac{1}{\Delta}\left\{\left(r^{2}+a^{2}\right) \frac{\partial}{\partial t}+a \frac{\partial}{\partial \varphi}-(r-M) s\right\}^{2}-4 s(r+i a \cos \vartheta) \frac{\partial}{\partial t} \\
& \left.+\frac{\partial}{\partial \cos \vartheta} \sin ^{2} \vartheta \frac{\partial}{\partial \cos \vartheta}+\frac{1}{\sin ^{2} \vartheta}\left\{a \sin ^{2} \vartheta \frac{\partial}{\partial t}+\frac{\partial}{\partial \varphi}+i s \cos \vartheta\right\}^{2}\right) \phi=0 .
\end{aligned}
$$

For $s=1$, this equation describes the first component $\Psi_{0}$ of the Newman-Penrose wave function $\left(\Psi_{0}, \Psi_{1}, \Psi_{2}\right)$ for electromagnetic waves, whereas the parameter value $s=-1$ gives the equation for $\Psi_{2}$. Likewise, setting $s=2$ gives the first component $\Psi_{0}$ of the Newman-Penrose wave function $\left(\Psi_{0}, \ldots, \Psi_{4}\right)$ for gravitational waves, whereas $s=-2$ gives the equation for $\Psi_{4}$. By direct inspection, one sees that setting $s=0$ gives back the scalar wave equation (5.3). We remark that setting $s=\frac{1}{2}$ gives the massless Dirac equation [43], and $s=\frac{3}{2}$ gives the massless Rarita-Schwinger equation [32].

We close with a remark on gravitational perturbations. As outlined above, our method is to consider perturbations of the Weyl tensor. Alternatively, one could consider perturbations of the metric (indeed, this was historically the first approach, going back to the stability analysis by Regge and Wheeler [40]). Working with metric perturbations has the disadvantage that infinitesimal coordinate transformations also lead to perturbations of the metric, which however have no geometric significance. In other words, when working with metric perturbations, the diffeomorphism invariance leads 
to a gauge freedom which is not easy to handle. This was the original motivation for Teukolsky and Press to consider instead perturbations of geometric quantities like the Newman-Penrose components of the Weyl tensor, leading to the Teukolsky framework. However, for some applications (for example in order to include matter models or to describe nonlinear waves) it is necessary to work with metric perturbations. Therefore, working in the Teukolsky formulation, the following question remains: Given a linear perturbation of the Weyl tensor, how can it be realized by a metric perturbation? This is an interesting and in general difficult question which we cannot analyze here (see however [46] and the references therein).

\section{Separation of the Teukolsky Equation}

The Teukolsky equation (6.6) has the remarkable property that it can be completely separated into a system of ordinary differential equations (ODEs): Due to the stationarity and axisymmetry, we can separate the $t$ - and $\varphi$-dependence with the usual plane-wave ansatz

$$
\phi(t, r, \vartheta, \varphi)=e^{-i \omega t-i k \varphi} \phi(r, \vartheta),
$$

where $\omega$ is a quantum number which could be real or complex and which corresponds to the "energy", and $k \in \mathbb{Z} / 2$ is a quantum number corresponding to the projection of angular momentum onto the axis of symmetry of the black hole (if $s$ is half an odd integer, then so is $k$ ). Substituting (7.1) into (6.6), we see that the Teukolsky operator splits into the sum of radial and angular parts, giving rise to the equation

$$
\left(\mathcal{R}_{\omega, k}+\mathcal{A}_{\omega, k}\right) \phi=0
$$

where $\mathcal{R}_{\omega, k}$ and $\mathcal{A}_{\omega, k}$ are given by (for details see [30, Section 6])

$$
\begin{aligned}
& \mathcal{R}_{\omega}=-\frac{\partial}{\partial r} \Delta \frac{\partial}{\partial r}-\frac{1}{\Delta}\left(\omega\left(r^{2}+a^{2}\right)+a k-i(r-M) s\right)^{2}-4 i s r \omega+4 k a \omega \\
& \mathcal{A}_{\omega}=-\frac{\partial}{\partial \cos \vartheta} \sin ^{2} \vartheta \frac{\partial}{\partial \cos \vartheta}+\frac{1}{\sin ^{2} \vartheta}\left(-a \omega \sin ^{2} \vartheta+k-s \cos \vartheta\right)^{2} .
\end{aligned}
$$

We can therefore separate the variables $r$ and $\vartheta$ with the multiplicative ansatz

$$
\phi(r, \vartheta)=R(r) \Theta(\vartheta),
$$

to obtain for given $\omega$ and $k$ the system of ODEs

$$
\mathcal{R}_{\omega, k} R_{\lambda}=-\lambda R_{\lambda}, \quad \mathcal{A}_{\omega, k} \Theta_{\lambda}=\lambda \Theta_{\lambda}
$$

Solutions of the coupled system (7.5) are referred to as mode solutions.

We point out that the last separation (7.4) is not obvious because it does not correspond to an underlying space-time symmetry. Instead, as discovered by Carter for the scalar wave equation [6], it corresponds to the fact that in the Kerr geometry there exists an irreducible quadratic Killing tensor (i.e. a Killing tensor which is not a symmetrized tensor product of Killing vectors). The separation constant $\lambda$ is an eigenvalue of the angular operator $\mathcal{A}_{\omega, k}$ and can thus be thought of as an angular quantum number. In the spherically symmetric case $a=0$, this separation constant goes over to the usual eigenvalues $\lambda=l(l+1)$ of the total angular momentum operator. 


\section{Results on Linear Stability and Superradiance}

Being familiar with the structure of the different linear wave equations, we can now state our results on stability and superradiance. The problem of linear stability of black holes amounts to the question whether solutions of the corresponding linear wave equations decay for large times. In order to put our results into context, we point out that the problem of linear stability of black holes has a long history. It goes back to the study of the Schwarzschild black hole by Regge and Wheeler [40] who showed that an integral norm of the perturbation of each angular mode is bounded uniformly in time. Decay of these perturbations was first proved in [31. More detailed estimates of metric perturbations in Schwarzschild were obtained in [9, 34]. For the Kerr black hole, linear stability under perturbations of general spin has been an open problem for many years, which was solved in the dynamical setting in [30] (for related results obtained with different methods see [35, 3, 2, 10] and the references in these papers). A key ingredient to our proof is the so-called mode stability result obtained by Whiting [45, who proved that the Teukolsky equation does not admit solutions which decay both at spatial infinity and at the event horizon and increase exponentially in time.

We consider the Cauchy problem for the Teukolsky equation. Thus we seek a solution $\phi$ of the Teukolsky equation (6.6) for given initial data

$$
\left.\phi\right|_{t=0}=\phi_{0} \quad \text { and }\left.\quad \partial_{t} \phi\right|_{t=0}=\phi_{1} .
$$

Being a linear hyperbolic PDE, the Cauchy problem for the Teukolsky equation has unique global solutions. Also, taking smooth initial data, the solution is smooth for all times. Our task is to show that solutions decay for large times. In order to avoid specifying decay assumptions at the event horizon and at spatial infinity, we restrict attention to compactly supported initial data outside the event horizon,

$$
\phi_{0}, \phi_{1} \in C_{0}^{\infty}\left(\left(r_{1}, \infty\right) \times S^{2}\right) .
$$

Since the Kerr geometry is axisymmetric, the Teukolsky equation decouples into separate equations for each azimuthal mode. Therefore, the solution of the Cauchy problem is obtained by solving the Cauchy problem for each azimuthal mode and taking the sum of the resulting solutions. With this in mind, we restrict attention to the Cauchy problem for a single azimuthal mode, i.e.

$$
\phi_{0}(r, \vartheta, \varphi)=e^{-i k \varphi} \phi_{0}^{(k)}(r, \vartheta), \quad \phi_{1}(r, \vartheta, \varphi)=e^{-i k \varphi} \phi_{1}^{(k)}(r, \vartheta)
$$

for given $k \in \mathbb{Z} / 2$. The main result of [30] is stated as follows:

Theorem 8.1. Consider a non-extreme Kerr black hole of mass $M$ and angular momentum $a M$ with $M^{2}>a^{2}>0$. Then for any $s \geq \frac{1}{2}$ and any $k \in \mathbb{Z} / 2$, the solution of the Teukolsky equation with initial data of the form (8.1) and (8.2) decays to zero in $L_{\mathrm{loc}}^{\infty}\left(\left(r_{1}, \infty\right) \times S^{2}\right)$.

This theorem establishes in the dynamical setting that the non-extreme Kerr black hole is linearly stable.

Our method of proof uses an integral representation of the time evolution operator involving the radial and angular solutions of the separated system of ODEs (7.5). Such an integral representation was derived earlier for the scalar wave equation in [17], and it was used for proving decay in time [18]. Moreover, in [19] it was proven in the dynamical setting that superradiance occurs for scalar waves. We now explain 
this result. Superradiance for scalar waves in the Kerr geometry was first studied by Zel'dovich and Starobinsky [47, 41] on the level of modes. More precisely, they computed the transmission and reflection coefficients for the radial ODE in (7.5). The absolute value squared of these coefficients can be interpreted as the energy flux of the incoming and outgoing waves, respectively. Comparing these fluxes, one obtains the relative energy gain. Starobinsky computed the relative gain of energy to about $5 \%$ for $k=1$ and less than $1 \%$ for $k \geq 2$.

Unfortunately, this mode analysis does not give information on the dynamics. Thus for a rigorous treatment of energy extraction one needs to consider the time-dependent situation. In [19], this is accomplished by constructing initial data of the form of wave packets, in such a way that the energy gain agrees with the results of the mode analysis up to an arbitrarily small error. The crucial analytical ingredient to the proof is the time-independent energy estimate for the outgoing wave as derived in [24].

The remainder of these lectures is devoted to giving an outline of the proof of Theorem 8.1. Before entering the constructions, we point out the main difficulties:

- The Teukolsky equation for $s \neq 0$ is not of variational form, i.e. it cannot be obtained as the Euler-Lagrange equation of an action.

- As a consequence, we cannot apply Noether's theorem to obtain conserved quantities. In particular, there is no conserved energy, being an integral of an energy density. This means that, in contrast to the situation described for the Dirac equation in Section 6.1, the time evolution cannot be described by a unitary operator on a Hilbert space. As a consequence, we cannot use the spectral theorem for selfadjoint or unitary operators on Hilbert spaces.

- A related difficulty is that the coefficients of the first derivative terms in the Teukolsky equation for $s \neq 0$ are complex. Such complex potentials in a wave equation usually describe dissipation, implying that (depending of the sign of the dissipation terms) the solutions typically decay or increase exponentially in time. This means that, in order to show that the solution of the Teukolsky equation decays for large times, one must carefully control the signs and the size of the complex coefficients by quantitative estimates.

- In the separation of variables (7.5), both the radial and angular differential operators $\mathcal{R}_{\omega, k}$ and $\mathcal{A}_{\omega, k}$ depend on the separation constants $k$ and $\omega$. As a consequence, it is not at all obvious if and how for given initial data one can decompose the corresponding solution of the Cauchy problem into a superposition of mode solutions. An obvious difficulty is that, for such a mode decomposition, one would have to know the separation constant $\omega$, which in turn can be specified only if we already know the full dynamics of the wave.

\section{Hamiltonian Formulation and Integral Representations}

In order to analyze the dynamics of the Teukolsky wave, it is useful to work with contour integrals of the resolvent of the Hamiltonian, as we now outline. In preparation, we must rewrite the Teukolsky equation in Hamiltonian form. To this end, we introduce the two-component wave function

$$
\Psi=\sqrt{r^{2}+a^{2}}\left(\begin{array}{c}
\phi \\
i \partial_{t} \phi
\end{array}\right)
$$

and write the Teukolsky equation as

$$
i \partial_{t} \Psi=H \Psi
$$


where $H$ is a second-order spatial differential operator. We consider $H$ as an operator on a Hilbert space $\mathcal{H}$ with the domain

$$
\mathcal{D}(H)=C_{0}^{\infty}\left(\left(r_{1}, \infty\right) \times S^{2}, \mathbb{C}^{4}\right) .
$$

It would be desirable to represent $H$ as a self-adjoint operator on a Hilbert space $\mathcal{H}$, because it would then be possible to apply the spectral calculus and write the time evolution operator similar as for the Dirac equation in the form (6.2). Unfortunately, this procedure does not work here, as can be understood as follows. As already mentioned at the end of the previous section, the Teukolsky equation is not of variational form, implying that there is no conserved energy. If there were a conserved bilinear form $\langle\Psi \mid \Phi\rangle$ on the solutions, then the calculation

$$
0=\partial_{t}\langle\Psi \mid \Phi\rangle=\langle\dot{\Psi} \mid \Phi\rangle+\langle\Psi \mid \dot{\Phi}\rangle=i(\langle H \Psi \mid \Phi\rangle-\langle\Psi \mid H \Phi\rangle)
$$

would imply that the Hamiltonian were symmetric with respect to this bilinear form. But, having no conserved energy, there is also no bilinear form with respect to which the Hamiltonian is symmetric. In order to avoid confusion, we remark that there is a conserved physical energy, which in the example of a Maxwell field could be written in the form (5.4) with $T_{i j}$ the energy-momentum tensor of the Maxwell field. However, this energy involves all the components of the field tensor or, in other words, all the components of the Newman-Penrose wave function in (6.3). Since the Teukolsky equation only gives $\Psi_{0}$ or $\Psi_{2}$, we would have to compute the other components using the Teukolsky-Starobinsky identities. As a consequence, the resulting formula for the Maxwell energy would involve higher derivatives of the Teukolsky wave function, making the situation very complicated. This is why we decided not to use the physical energy in our construction.

We conclude that we shall treat the operator $H$ as a non-symmetric operator on a Hilbert space. In order to get an idea for how to work with non-symmetric operators, it is helpful get a motivation from the finite-dimensional setting. Thus let $A$ be a linear operator on a finite-dimensional Hilbert space $\mathcal{H}$. Clearly, this operator need not be diagonalizable, because Jordan chains may form. Nevertheless, one can get a spectral calculus by working with contour integrals:

Lemma 9.1. Let $A$ be a linear operator $A$ on a Hilbert space $\mathcal{H}$ of dimension $n<\infty$. Then

$$
e^{-i t A}=-\frac{1}{2 \pi i} \oint_{\Gamma} e^{-i \omega t}(A-\omega)^{-1} d \omega
$$

where $\Gamma$ is a contour which encloses the whole spectrum of $A$ with winding number one.

Proof. If $A$ is diagonalizable, we can choose a basis where $A$ is diagonal,

$$
A=\operatorname{diag}\left(\lambda_{1}, \ldots, \lambda_{n}\right) .
$$

In this case, (9.2) is obtained immediately by carrying out the contour integral for each matrix entry with the help of the Cauchy integral formula.

The case that $A$ is not diagonalizable can be obtained by approximation, noting that the diagonalizable matrices are dense and that both sides of (9.2) are continuous on the space of matrices (endowed with the topology of $\mathbb{C}^{n \cdot n}$ ).

Motivated by this formula for matrices, we can hope that the Cauchy problem for the equation (9.1) with initial data $\Psi_{0}$ could be solved with the Cauchy integral formula 
by

$$
\Psi(t)=-\frac{1}{2 \pi i} \oint_{\Gamma} e^{-i \omega t}(H-\omega)^{-1} \Psi_{0} d \omega
$$

where $\Gamma$ is a contour which encloses all eigenvalues of $H$ (note that this formula holds for any matrix $H$, even if it is not diagonalizable). It turns out that in our infinitedimensional setting, this formula indeed holds. The first step in making sense of this formula is to localize the spectrum of $H$ and to make sure that the resolvent exists along the integration contour. To this end, we choose the scalar product on $\mathcal{H}$ as a suitable weighted Sobolev scalar product in such a way that that the operator $H-H^{*}$ is bounded, i.e.

$$
\left\|H-H^{*}\right\| \leq \frac{c}{2}
$$

with a suitable constant $c>0$. Then we prove that the resolvent $R_{\omega}:=(H-\omega)^{-1}$ exists if $\omega$ lies outside a strip enclosing the real axis (see [30, Lemma 4.1]):

Lemma 9.2. For every $\omega$ with

$$
|\operatorname{Im} \omega|>c,
$$

the resolvent $R_{\omega}=(H-\omega)^{-1}$ exists and is bounded by

$$
\left\|R_{\omega}\right\| \leq \frac{1}{|\operatorname{Im} \omega|-c} .
$$

When forming contour integrals, one must always make sure to stay outside the strip $|\operatorname{Im} \omega| \leq c$, making it impossible to work with closed contours enclosing the spectrum. But we can work with unbounded contours as follows (see [30, Corollary 5.3]):

Proposition 9.3. For any integer $p \geq 1$, the solution of the Cauchy problem for the Teukolsky equation with initial data $\left.\Psi\right|_{t=0}=\Psi_{0} \in \mathcal{D}(H)$ has the representation

$$
\Psi(t)=-\frac{1}{2 \pi i} \int_{C} e^{-i \omega t} \frac{1}{(\omega+3 i c)^{p}}\left(R_{\omega}(H+3 i c)^{p} \Psi_{0}\right) d \omega,
$$

where $C$ is the contour

$$
C=\{\omega \mid \operatorname{Im} \omega=2 c\} \cup\{\omega \mid \operatorname{Im} \omega=-2 c\}
$$

with counter-clockwise orientation.

Here the factor $(\omega+3 i c)^{-p}$ gives suitable decay for large $|\omega|$ and ensures that the integral converges in the Hilbert space $\mathcal{H}$.

The representation (9.4) gives an explicit solution of the Cauchy problem in terms of a Cauchy integral of the resolvent. Unfortunately, this representation does not immediately give information on the long-time dynamics of the Teukolsky wave. This shortcoming can be understood immediately from the fact that the factor $e^{-i \omega t}$ in the integrand increases exponentially for large times because $\left|e^{-i \omega t}\right|=e^{\operatorname{Im} \omega t}=e^{ \pm 2 c t}$. In order to bypass this shortcoming, our strategy is to move the contour onto the real axis. Once this has been accomplished, the integral representation (9.4) simplifies to a Fourier transform,

$$
\Psi(t)=\int_{-\infty}^{\infty} e^{-i \omega t} \hat{\Psi}(\omega) d \omega
$$

The decay of such a Fourier transform can be obtained from the Riemann-Lebesgue lemma, stating that

$$
\hat{\Psi} \in L^{1}(\mathbb{R}, d \omega) \quad \Longrightarrow \quad \lim _{t \rightarrow \pm \infty} \Psi(t)=0
$$


(where the wave functions are evaluated pointwise in space). One of the difficulties in making this strategy work is to prove that the contour can indeed be moved onto the real axis. This makes it necessary to show that the Hamiltonian has no spectrum away from the real axis. We did not succeed in proving this result using operator theoretic methods. Instead, our method is to first make use of the separation of variables, making it possible rule out the spectrum in the complex plane using Whiting's mode stability result [45].

\section{A Spectral Decomposition of the Angular Teukolsky Operator}

Following the strategy we just outlined, our next task is to employ the separation of variables in the integrand of the integral representation (9.4). Regarding the angular equation (7.5) as an eigenvalue equation, we are led to considering the angular operator $\mathcal{A}_{\omega}$ in (7.3) as an operator on the Hilbert space

$$
\mathcal{H}_{k}:=L^{2}\left(S^{2}\right) \cap\left\{e^{-i k \varphi} \Theta(\vartheta) \mid \Theta:(0, \pi) \rightarrow \mathbb{C}\right\}
$$

with dense domain $\mathcal{D}\left(\mathcal{A}_{\omega}\right)=C^{\infty}\left(S^{2}\right) \cap \mathcal{H}_{k}$. Unfortunately, the parameter $\omega$ is not real but lies on the contour (9.5). As a consequence, the operator $\mathcal{A}_{\omega}$ is not symmetric, because its adjoint is given by

$$
\mathcal{A}_{\omega}^{*}=\mathcal{A}_{\bar{\omega}} \neq \mathcal{A}_{\omega} .
$$

The operator $\mathcal{A}_{\omega}$ is not even a normal operator, making it impossible to apply the spectral theorem in Hilbert spaces. Indeed, $\mathcal{A}_{\omega}$ does not need to be diagonalizable, because there might be Jordan chains. On the other hand, in order to make use of the separation of variables, we must decompose the initial data into angular modes. This can be achieved by decomposing the angular operator into invariant subspaces of bounded dimension, as is made precise in the following theorem (see [28, Theorem 1.1]):

Theorem 10.1. Let $U \subset \mathbb{C}$ be the strip

$$
|\operatorname{Im} \omega|<3 c .
$$

Then there is a positive integer $N$ and a family of bounded linear operators $Q_{n}^{\omega}$ on $\mathcal{H}_{k}$ defined for all $n \in \mathbb{N} \cup\{0\}$ and $\omega \in U$ with the following properties:

(i) The image of the operator $Q_{0}^{\omega}$ is an $N$-dimensional invariant subspace of $\mathcal{A}_{k}$.

(ii) For every $n \geq 1$, the image of the operator $Q_{n}^{\omega}$ is an at most two-dimensional invariant subspace of $\mathcal{A}_{k}$.

(iii) The $Q_{n}^{\omega}$ are uniformly bounded in $\mathrm{L}\left(\mathcal{H}_{k}\right)$, i.e. for all $n \in \mathbb{N} \cup\{0\}$ and $\omega \in U$,

$$
\left\|Q_{n}^{\omega}\right\| \leq c_{2}
$$

for a suitable constant $c_{2}=c_{2}(s, k, c)$ (here $\|\cdot\|$ denotes the sup-norm on $\mathcal{H}_{k}$ ).

(iv) The $Q_{n}^{\omega}$ are idempotent and mutually orthogonal in the sense that

$$
Q_{n}^{\omega} Q_{n^{\prime}}^{\omega}=\delta_{n, n^{\prime}} Q_{n}^{\omega} \quad \text { for all } n, n^{\prime} \in \mathbb{N} \cup\{0\} .
$$

(v) The $Q_{n}^{\omega}$ are complete in the sense that for every $\omega \in U$,

$$
\sum_{n=0}^{\infty} Q_{n}^{\omega}=\mathbb{1}
$$

with strong convergence of the series. 


\section{Invariant Disk Estimates for the Complex Riccati Equation}

In order to locate the spectrum of $\mathcal{A}_{\omega}$, we use detailed ODE estimates. The operators $Q_{n}^{\omega}$ are then obtained similar to (9.3) as Cauchy integrals,

$$
Q_{n}^{\omega}:=-\frac{1}{2 \pi i} \oint_{\Gamma_{n}} s_{\lambda} d \lambda, \quad n \in \mathbb{N}_{0},
$$

where the contour $\Gamma_{n}$ encloses the corresponding spectral points, and $s_{\lambda}=\left(\mathcal{A}_{\omega}-\lambda\right)^{-1}$ is the resolvent of the angular operator. What makes the analysis doable is the fact that $\mathcal{A}_{\omega}$ is an ordinary differential operator. Transforming the angular equation in (7.5) into Sturm-Liouville form

$$
\left(-\frac{d^{2}}{d u^{2}}+V(u)\right) \phi=0
$$

(where $u=\vartheta$ and $V \in C^{\infty}((0, \pi), \mathbb{C})$ is a complex potential), the resolvent $s_{\lambda}$ can be represented as an integral operator whose kernel is given explicitly in terms of suitable fundamental solutions $\phi_{L}^{\mathrm{D}}$ and $\phi_{R}^{\mathrm{D}}$,

$$
s_{\lambda}\left(u, u^{\prime}\right)=\frac{1}{w\left(\phi_{L}^{\mathrm{D}}, \phi_{R}^{\mathrm{D}}\right)} \times \begin{cases}\phi_{L}^{\mathrm{D}}(u) \phi_{R}^{\mathrm{D}}\left(u^{\prime}\right) & \text { if } u \leq u^{\prime} \\ \phi_{L}^{\mathrm{D}}\left(u^{\prime}\right) \phi_{R}^{\mathrm{D}}(u) & \text { if } u^{\prime}<u,\end{cases}
$$

where $w\left(\phi_{L}^{\mathrm{D}}, \phi_{R}^{\mathrm{D}}\right)$ denotes the Wronskian.

The main task is to find good approximations for the solutions of the Sturm-Liouville equation (11.1) with rigorous error bounds which must be uniform in the parameters $\omega$ and $\lambda$. These approximations are obtained by "glueing together" suitable WKB, Airy and parabolic cylinder functions. The needed properties of these special functions are derived in [26]. In order to obtain error estimates, we combine several methods:

(a) Osculating circle estimates (see [28, Section 6])

(b) The $T$-method (see [27, Section 3.2])

(c) The $\kappa$-method (see [27, Section 3.3])

The method (a) is needed in order to separate the spectral points of $\mathcal{A}_{\omega}$ (gap estimates). The methods (b) and (c) are particular versions of invariant disk estimates as derived for complex potentials in [25] (based on previous estimates for real potentials in [23] and [18]). These estimates are also needed for the analysis of the radial equation, see Section 12 below. We now explain the basic idea behind the invariant disk estimates.

Let $\phi$ be a solution of the Sturm-Liouville equation (11.1) with a complex potential $V$. Then the function $y$ defined by

$$
y=\frac{\phi^{\prime}}{\phi}
$$

is a solution of the Riccati equation

$$
y^{\prime}=V-y^{2} .
$$

Conversely, given a solution $y$ of the Riccati equation, a corresponding fundamental system for the Sturm-Liouville equation is obtained by integration. With this in mind, it suffices to construct a particular approximate solution $\tilde{y}$ and to derive rigorous error estimates. The invariant disk estimates are based on the observation that the Riccati flow maps disks to disks (see [25, Sections 2 and 3]). In fact, denoting the center of 
the disk by $m \in \mathbb{C}$ and its radius by $R>0$, we get the flow equations

$$
\begin{aligned}
R^{\prime} & =-2 R \operatorname{Re} m \\
m^{\prime} & =V-m^{2}-R^{2} .
\end{aligned}
$$

Clearly, this system of equations is as difficult to solve as the original Riccati equation (11.3). But suppose that $m$ is an approximate solution in the sense that

$$
\begin{aligned}
R^{\prime} & =-2 R \operatorname{Re} m+\delta R \\
m^{\prime} & =V-m^{2}-R^{2}+\delta m,
\end{aligned}
$$

with suitable error terms $\delta m$ and $\delta R$, then the Riccati flow will remain inside the disk provided that its radius grows sufficiently fast, i.e. (see [25, Lemma 3.1])

$$
\delta R \geq|\delta m| .
$$

This is the starting point for the invariant disk method. In order to reduce the number of free functions, it is useful to solve the linear equations in the above system of ODEs by integration. For more details we refer the reader to [25, 27].

\section{Separation of the Resolvent and Contour Deformations}

The next step is to use the spectral decomposition of the angular operator in Theorem 10.1 in the integral representation of the solution of the Cauchy problem. More specifically, inserting (10.1) into (9.4) gives

$$
\Psi(t)=-\frac{1}{2 \pi i} \int_{C} \sum_{n=0}^{\infty} e^{-i \omega t} \frac{1}{(\omega+3 i c)^{p}}\left(R_{\omega} Q_{n}^{\omega}(H+3 i c)^{p} \Psi_{0}\right) d \omega .
$$

At this point, the operator product $R_{\omega} Q_{n}^{\omega}$ can be expressed in terms of solutions of the radial and angular ODEs (7.5) which arise in the separation of variables (see 30, Theorem 7.1]). Namely, the operator $Q_{\omega}^{n}$ maps onto an invariant subspace of $\mathcal{A}_{\omega}$ of dimension at most $N$, and it turns out that the operator product $R_{\omega} Q_{n}^{\omega}$ leaves this subspace invariant. Therefore, choosing a basis of this invariant subspace, the PDE $(H-\omega) R_{\omega} Q_{\omega}^{n}=Q_{\omega}^{n}$ can be rewritten as a radial ODE involving matrices of rank at most $N$. The solution of this ODE can be expressed explicitly in terms of the resolvent of the radial ODE. In order to compute this resolvent, it is useful to also transform the radial ODE into Sturm-Liouville form (11.1). To this end, we introduce the Regge-Wheeler coordinate $u \in \mathbb{R}$ by

$$
\frac{d u}{d r}=\frac{r^{2}+a^{2}}{\Delta}
$$

mapping the event horizon to $u=-\infty$. Then the radial ODE takes again the form (11.1), but now with $u$ defined on the whole real axis. Thus the resolvent can be written as an integral operator with kernel given in analogy to (11.2) by

$$
s_{\omega}(u, v)=\frac{1}{w(\grave{\phi}, \grave{\phi})} \times \begin{cases}\dot{\phi}(u) \grave{\phi}(v) & \text { if } v \geq u \\ \grave{\phi}(u) \dot{\phi}(v) & \text { if } v<u,\end{cases}
$$

where $\dot{\phi}$ and $\grave{\phi}$ form a specific fundamental system for the radial ODE. The solutions $\dot{\phi}$ and $\grave{\phi}$ are constructed as Jost solutions, using methods of one-dimensional scattering theory (see [11] and [30, Section 6], [18, Section 3]).

The next step is to deform the contour in the integral representation (12.1). Standard arguments show that the integrand in (12.1) is holomorphic on the resolvent set 
(i.e. for all $\omega$ for which the resolvent $R_{\omega}$ in (9.4) exists). Thus the contour may be deformed as long as it does not cross singularities of the resolvent. Therefore, it is crucial to show that the integrand in (12.1) is meromorphic and to determine its pole structure. Here we make essential use of Whiting's mode stability result [45] which states, in our context, that every summand in (12.1) is holomorphic off the real axis. In order to make use of this mode stability, we need to interchange the integral in (12.1) with the infinite sum. To this end, we derive estimates which show that the summands in (12.1) decay for large $n$ uniformly in $\omega$. Here we again use ODE techniques, in the same spirit as described above for the angular equation (see [30, Section 10]). In this way, we can move the contour in the lower half plane arbitrarily close to the real axis. Moreover, the contour in the upper half plane may be moved to infinity. We thus obtain the integral representation (see [30, Corollary 10.4])

$$
\Psi(t)=-\frac{1}{2 \pi i} \sum_{n=0}^{\infty} \lim _{\varepsilon \searrow 0} \int_{\mathbb{R}-i \varepsilon} \frac{e^{-i \omega t}}{(\omega+3 i c)^{p}}\left(R_{\omega, n} Q_{n}^{\omega}(H+3 i c)^{p} \Psi_{0}\right) d \omega .
$$

The remaining issue is that the integrands in this representation might have poles on the real axis. These so-called radiant modes are ruled out by a causality argument (see [30, Section 11]; for an alternative proof see [4]). We thus obtain the following result (see [30, Theorem 12.1]).

Theorem 12.1. For any $k \in \mathbb{Z} / 2$, there is a parameter $p>0$ such that for any $t<0$, the solution of the Cauchy problem for the Teukolsky equation with initial data

$$
\left.\Psi\right|_{t=0}=e^{-i k \varphi} \Psi_{0}^{(k)}(r, \vartheta) \quad \text { with } \quad \Psi_{0}^{(k)} \in C^{\infty}\left(\mathbb{R} \times S^{2}, \mathbb{C}^{2}\right)
$$

has the integral representation

$$
\begin{aligned}
& \Psi(t, u, \vartheta, \varphi) \\
& \quad=-\frac{1}{2 \pi i} e^{-i k \varphi} \sum_{n=0}^{\infty} \int_{-\infty}^{\infty} \frac{e^{-i \omega t}}{(\omega+3 i c)^{p}}\left(R_{\omega, n}^{-} Q_{n}^{\omega}(H+3 i c)^{p} \Psi_{0}^{(k)}\right)(u, \vartheta) d \omega,
\end{aligned}
$$

where $R_{\omega, n}^{-} \Psi:=\lim _{\varepsilon \searrow 0}\left(R_{\omega-i \varepsilon, n} \Psi\right)$. Moreover, the integrals in (12.2) all exist in the Lebesgue sense. Furthermore, for every $\varepsilon>0$ and $u_{\infty} \in \mathbb{R}$, there is $N$ such that for all $u<u_{\infty}$

$$
\sum_{n=N}^{\infty} \int_{-\infty}^{\infty}\left\|\frac{1}{(\omega+3 i c)^{p}}\left(R_{\omega, n}^{-} Q_{n}^{\omega}(H+3 i c)^{p} \Psi_{0}^{(k)}\right)(u)\right\|_{L^{2}\left(S^{2}\right)} d \omega<\varepsilon .
$$

\section{Proof of Pointwise Decay}

Theorem 8.1 is a direct consequence of the integral representation (12.2) in Theorem 12.1. Namely, combining the estimate (12.3) with Sobolev methods, one can make the contributions for large $n$ pointwise arbitrarily small. On the other hand, for each of the angular modes $n=0, \ldots, N-1$, the desired pointwise decay as $t \rightarrow-\infty$ follows from the Riemann-Lebesgue lemma. For details we refer to [30, Section 12].

\section{Concluding Remarks}

We first point out that the integral representation of Theorem 12.1 is a suitable starting point for a detailed analysis for the dynamics of the solutions of the Teukolsky equation. In particular, one can study decay rates (similar as worked out for massive 
Dirac waves in [18]) and derive uniform energy estimates outside the ergosphere (similar as for scalar waves in [24]). Moreover, using the methods in [19], one could analyze superradiance phenomena for wave packets in the time-dependent setting.

Clearly, the next challenge is to prove nonlinear stability of the Kerr geometry. This will make it necessary to refine our results on the linear problem, for example by deriving weighted Sobolev estimates and by analyzing the $k$-dependence of our estimates. Moreover, it might be useful to combine our methods and results with microlocal techniques (as used for example in the proof of nonlinear stability results in the related Kerr-De Sitter geometry [33]).

Acknowledgments: I would like to thank the organizers of the first "Domoschool International Alpine School of Mathematics and Physics" held in Domodossola, 16-20 July 2018, for the kind invitation. This article is based on my lectures delivered at this summer school. I am grateful to Niky Kamran and Igor Khavkine for helpful comments on the manuscript.

\section{REFERENCES}

[1] R. Adler, M. Bazin, and M. Schiffer, Introduction to General Relativity, McGraw-Hill Book Co., New York-Toronto-London-Sydney, 1965.

[2] L. Andersson, T. Bäckdahl, and P. Blue, A new tensorial conservation law for Maxwell fields on the Kerr background, arXiv:1412.2960 [gr-qc], J. Differential Geom. 105 (2017), no. 2, 163-176.

[3] L. Andersson and P. Blue, Uniform energy bound and asymptotics for the Maxwell field on a slowly rotating Kerr black hole exterior, arXiv:1310.2664 [math.AP], J. Hyperbolic Differ. Equ. 12 (2015), no. 4, 689-743.

[4] L. Andersson, S. Ma, C. Paganini, and B.F. Whiting, Mode stability on the real axis, arXiv:1607.02759 [gr-qc], J. Math. Phys. 58 (2017), no. 7, 072501, 19.

[5] V. Cardoso, Ó.J.C. Dias, J.P.S. Lemos, and S. Yoshida, Black-hole bomb and superradiant instabilities, hep-th/0404096, Phys. Rev. D (3) 70 (2004), no. 4, 044039, 9.

[6] B. Carter, Republication of: Black hole equilibrium states. Part I. Analytic and geometric properties of the Kerr solutions, Gen. Relativity Gravitation 41 (2009), no. 12, 2873-2938.

[7] S. Chandrasekhar, The Mathematical Theory of Black Holes, Oxford Classic Texts in the Physical Sciences, The Clarendon Press Oxford University Press, New York, 1998.

[8] P.R. Chernoff, Essential self-adjointness of powers of generators of hyperbolic equations, J. Functional Analysis 12 (1973), 401-414.

[9] M. Dafermos, G. Holzegel, and I. Rodnianski, The linear stability of the Schwarzschild solution to gravitational perturbations, arXiv:1601.06467 [gr-qc] (2016).

[10] B Boundedness and decay for the Teukolsky equation on Kerr spacetimes I: the case $|a| \ll$ $m$, arXiv:1711.07944 [gr-qc] (2017).

[11] V. de Alfaro and T. Regge, Potential scattering, North-Holland Publishing Company, Amsterdam, 1965.

[12] F. Finster, Local U(2,2) symmetry in relativistic quantum mechanics, arXiv:hep-th/9703083, J. Math. Phys. 39 (1998), no. 12, 6276-6290.

[13] - The Continuum Limit of Causal Fermion Systems, arXiv:1605.04742 [math-ph], Fundamental Theories of Physics, vol. 186, Springer, 2016.

[14] F. Finster, N. Kamran, J. Smoller, and S.-T. Yau, Nonexistence of time-periodic solutions of the Dirac equation in an axisymmetric black hole geometry, gr-qc/9905047, Comm. Pure Appl. Math. 53 (2000), no. 7, 902-929.

[15] Decay rates and probability estimates for massive Dirac particles in the Kerr-Newman black hole geometry, arXiv:gr-qc/0107094, Commun. Math. Phys. 230 (2002), no. 2, 201-244.

[16] _ The long-time dynamics of Dirac particles in the Kerr-Newman black hole geometry, arXiv:gr-qc/0005088, Adv. Theor. Math. Phys. 7 (2003), no. 1, 25-52.

[17] An integral spectral representation of the propagator for the wave equation in the Kerr geometry, gr-qc/0310024, Commun. Math. Phys. 260 (2005), no. 2, 257-298. 
[18] Decay of solutions of the wave equation in the Kerr geometry, gr-qc/0504047, Commun. Math. Phys. 264 (2006), no. 2, 465-503.

[19] A rigorous treatment of energy extraction from a rotating black hole, arXiv:gr-qc/0701018, Commun. Math. Phys. 287 (2009), no. 3, 829-847.

[20] F. Finster and J. Kleiner, Causal fermion systems as a candidate for a unified physical theory, arXiv:1502.03587 [math-ph], J. Phys.: Conf. Ser. 626 (2015), 012020.

[21] F. Finster, J. Kleiner, and J.-H. Treude, An Introduction to the Fermionic Projector and Causal Fermion Systems, in preparation.

[22] F. Finster and C. Röken, Self-adjointness of the Dirac Hamiltonian for a class of non-uniformly elliptic boundary value problems, arXiv:1512.00761 [math-ph], Annals of Mathematical Sciences and Applications 1 (2016), no. 2, 301-320.

[23] F. Finster and H. Schmid, Spectral estimates and non-selfadjoint perturbations of spheroidal wave operators, J. Reine Angew. Math. 601 (2006), 71-107.

[24] F. Finster and J. Smoller, A time-independent energy estimate for outgoing scalar waves in the Kerr geometry, arXiv:0707.2290 [math-ph], J. Hyperbolic Differ. Equ. 5 (2008), no. 1, 221-255.

[25] Error estimates for approximate solutions of the Riccati equation with real or complex potentials, arXiv:0807.4406 [math-ph], Arch. Ration. Mech. Anal. 197 (2010), no. 3, 985-1009.

[26] - Absence of zeros and asymptotic error estimates for Airy and parabolic cylinder functions, arXiv:1207.6861 [math.CA], Commun. Math. Sci. 12 (2014), no. 1, 175-200.

[27] Refined error estimates for the Riccati equation with applications to the angular Teukolsky equation, arXiv:1307.6470 [math.CA], Methods Appl. Anal. 22 (2015), no. 1, 67-100.

[28] — A spectral representation for spin-weighted spheroidal wave operators with complex aspherical parameter, arXiv:1507.05756 [math-ph], Methods Appl. Anal. 23 (2016), no. 1, 35-118.

[29] L Linear stability of rotating black holes: Outline of the proof, Nonlinear Analysis in Geometry and Applied Mathematics (L. Bieri, P.T. Chruściel, and S.-T. Yau, eds.), arXiv:1609.03171 [math-ph], Harvard University Center of Mathematical Sciences and Applications (CMSA) Series in Mathematics, no. 1, International Press, Somerville, MA, 2017, pp. 77-90.

[30] Linear stability of the non-extreme Kerr black hole, arXiv:1606.08005 [math-ph], Adv. Theor. Math. Phys. 21 (2017), no. 8, 1991-2085.

[31] J.L. Friedman and M.S. Morris, Schwarzschild perturbations die in time, J. Math. Phys. 41 (2000), no. 11, 7529-7534.

[32] R. Güven, Black holes have no superhair, Phys. Rev. D 22 (1980), 2327-2330.

[33] P. Hintz and A. Vasy, The global non-linear stability of the Kerr-de Sitter family of black holes, arXiv:1606.04014 [math.DG], Acta Math. 220 (2018), no. 1, 1-206.

[34] P.-K. Hung, J. Keller, and M.-T. Wang, Linear stability of Schwarzschild spacetime: The Cauchy problem of metric coefficients, arXiv:1702.02843 [gr-qc] (2017).

[35] J. Metcalfe, D. Tataru, and M. Tohaneanu, Pointwise decay for the Maxwell field on black hole space-times, arXiv:1411.3693 [math.AP], Adv. Math. 316 (2017), 53-93.

[36] C.W. Misner, K.S. Thorne, and J.A. Wheeler, Gravitation, W.H. Freeman and Co., San Francisco, Calif., 1973.

[37] B. O'Neill, The Geometry of Kerr Black Holes, A K Peters Ltd., Wellesley, MA, 1995.

[38] R. Penrose, Gravitational collapse: The role of general relativity, Rev. del Nuovo Cimento 1 (1969), 252-276.

[39] W.H. Press and S.A. Teukolsky, Floating orbits, superradiant scattering and the black-hole bomb, Nature 238 (1972), 211-212.

[40] T. Regge and J.A. Wheeler, Stability of a Schwarzschild singularity, Phys. Rev. (2) 108 (1957), 1063-1069.

[41] A.A. Starobinsky, Amplification of waves during reflection from a black hole, Soviet Physics JETP 37 (1973), 28-32.

[42] N. Straumann, General Relativity, second ed., Graduate Texts in Physics, Springer, Dordrecht, 2013.

[43] S.A. Teukolsky, Perturbations of a rotating black hole I. Fundamental equations for gravitational, electromagnetic, and neutrino-field perturbations, Astrophys. J. 185 (1973), 635-647.

[44] R.M. Wald, General Relativity, University of Chicago Press, Chicago, IL, 1984.

[45] B.F. Whiting, Mode stability of the Kerr black hole, J. Math. Phys. 30 (1989), no. 6, 1301-1305. 
[46] B.F. Whiting and L.R. Price, Metric reconstruction from Weyl scalars, Classical Quantum Gravity 22 (2005), no. 15, S589-S604.

[47] Y.B. Zel'dovich, Amplification of cylindrical electromagnetic waves from a rotating body, Soviet Physics JETP 35 (1972), 1085-1087.

Fakultät Für Mathematik, Universität Regensburg, D-93040 Regensburg, Germany

E-mail address: finster@ur.de 